\title{
Excitations in a Four-Leg Antiferromagnetic Heisenberg Spin Tube
}

\author{
V. O. Garlea, ${ }^{1, *}$ A. Zheludev, ${ }^{1}$ L.-P. Regnault, ${ }^{2}$ J.-H. Chung, ${ }^{3, \dagger}$ Y. Qiu, ${ }^{3}$ M. Boehm, ${ }^{4}$ K. Habicht, ${ }^{5}$ and M. Meissner ${ }^{5}$ \\ ${ }^{1}$ Neutron Scattering Sciences Division, Oak Ridge National Laboratory, Oak Ridge, Tennessee 37831, USA \\ ${ }^{2}$ CEA-Grenoble, DRFMC-SPSMS-MDN, 17 rue des Martyrs, 38054 Grenoble Cedex 9, France \\ ${ }^{3}$ NCNR, National Institute of Standards and Technology, Gaithersburg, Maryland 20899, \\ and University of Maryland, College Park, Maryland, 20742, USA \\ ${ }^{4}$ Institut Laue Langevin, 6 rue J. Horowitz, 38042 Grenoble Cedex 9, France \\ ${ }^{5}$ BENSC, Hahn-Meitner Institut, D-14109 Berlin, Germany \\ (Received 3 October 2007; published 25 January 2008)
}

\begin{abstract}
Inelastic neutron scattering is used to investigate magnetic excitations in the quasi-one-dimensional quantum spin-liquid system $\mathrm{Cu}_{2} \mathrm{Cl}_{4} \cdot \mathrm{D}_{8} \mathrm{C}_{4} \mathrm{SO}_{2}$. Contrary to previously conjectured models that relied on bond-alternating nearest-neighbor interactions in the spin chains, the dominant interactions are actually next-nearest-neighbor in-chain antiferromagnetic couplings. The appropriate Heisenberg Hamiltonian is equivalent to that of a $S=1 / 2$ 4-leg spin-tube with almost perfect one dimensionality and no bond alternation. A partial geometric frustration of rung interactions induces a small incommensurability of short-range spin correlations.
\end{abstract}

DOI: 10.1103/PhysRevLett.100.037206

PACS numbers: 75.10.Jm, 75.50.Ee, 78.70.Nx

The $S=1 / 2$ antiferromagnetic (AF) Heisenberg spin ladder with an even number of legs is the cornerstone model in low-dimensional quantum magnetism [1-4]. Its magnetic long range order is totally destroyed by zeropoint quantum fluctuations, yet the "spin liquid" ground state is not entirely structureless, due to hidden topological nonlocal "string" correlations [5,6]. Extensive theoretical studies lead to a profound understanding of spin ladders and related models, including the origin of the energy gap [1-3], multiparticle continua and bound states [4,7], and the unique gapless quantum-critical phase that can be induced by external magnetic fields [8,9]. Only a handful of experimental realizations of the ladder model have been found to date. Unfortunately, while some of these, like IPA- $\mathrm{CuCl}_{3}[10,11]$, are not perfectly one-dimensional, others, like ( $\mathrm{La}, \mathrm{Ca}, \mathrm{Sr})_{14} \mathrm{Cu}_{24} \mathrm{O}_{41}$ [12,13], have energy scales and phase diagrams that can not be accessed in an experiment. The search for new prototype materials continues.

One compound recently discussed in this context is $\mathrm{Cu}_{2} \mathrm{Cl}_{4} \cdot \mathrm{D}_{8} \mathrm{C}_{4} \mathrm{SO}_{2}\left(\mathrm{Sul}_{-} \mathrm{Cu}_{2} \mathrm{Cl}_{4}\right)$. It has a conveniently small spin gap $\Delta \approx 0.5 \mathrm{meV}$ and was described as being composed of $S=1 / 2$ double-chains with possible geometrically frustrated diagonal rung interactions [14,15]. However, the gap in Sul- $\mathrm{Cu}_{2} \mathrm{Cl}_{4}$ could be fully accounted for without invoking the rung interactions, [14] by a slight structural alternation in the bond lengths. The corresponding bond-alternating $S=1 / 2$ chain can be viewed as an array of interacting $\mathrm{AF}$ spin dimers, like those found in $\mathrm{CuGeO}_{3}[16,17]$ and $\mathrm{Cu}\left(\mathrm{NO}_{3}\right)_{2} \cdot 2.5 \mathrm{D}_{2} \mathrm{O}$ [18]. Systems of this type realize a simple "local" spin liquid ground state that lacks the hidden symmetry violation or the translational invariance of the ladder model. Below, we present the results of inelastic neutron scattering experiments that reveal that $\mathrm{Sul}-\mathrm{Cu}_{2} \mathrm{Cl}_{4}$ is actually not a spin-dimer material, but neither it is a triangular ladder. Its key magnetic interactions lead to the formation of uniform 4-leg spin tubes with no bond alternation and almost perfect 1D character. A geometric frustration stabilizes unique incommensurate dynamic spin correlations.

Sul- $\mathrm{Cu}_{2} \mathrm{Cl}_{4}$ crystallizes in a triclinic space group $P \overline{1}$ with $a=9.42 \AA, b=10.79 \AA, c=6.62 \AA, \alpha=98.87^{\circ}$, $\beta=95.25^{\circ}$, and $\gamma=120.85^{\circ}$ [14]. Pairs of $S=1 / 2$ chains built of edge-sharing $\mathrm{CuCl}_{5} \mathrm{O}$ octahedra run along the crystallographic $c$-axis (Fig. 1). As mentioned above, due to the presence of two nonequivalent $\mathrm{Cu}^{2+}$ sites, there is a slight alternation (about $0.5 \%$ ) in the distance between consecutive magnetic ions, $\mathrm{Cu}_{1}$ and $\mathrm{Cu}_{2}$. In the original model of Ref. [14], these chains form the spin ladder legs, while the rungs involve nearby $\mathrm{Cu}^{2+}$ cations packed along the $b$ axis. Both rung and leg magnetic interactions are

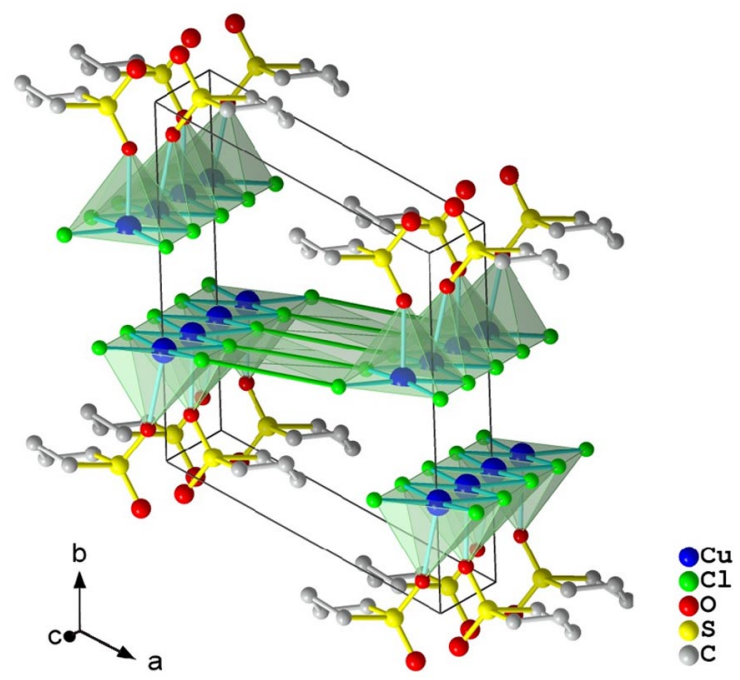

FIG. 1 (color online). Schematic view of the crystal structure of Sul- $\mathrm{Cu}_{2} \mathrm{Cl}_{4}$. Dominant AF interactions between $S=1 / 2$ $\mathrm{Cu}^{2+}$ ions are mediated by $\mathrm{Cu}-\mathrm{Cl}-\mathrm{Cl}-\mathrm{Cu}$ superexchange bridges. 
established via $\mathrm{Cu}-\mathrm{Cl}-\mathrm{Cu}$ superexchange pathways. A key feature of this model is that the distance between consecutive spins on each leg is $c / 2$, so that the $1 \mathrm{D}$ AF zonecenters are located at integer values of $l$, where $(h, k, l)$ are wave vector's reciprocal coordinates.

Previous neutron scattering studies employed thermal neutrons and protonated Sul- $\mathrm{Cu}_{2} \mathrm{Cl}_{4}$ samples [19], but failed to detect any magnetic scattering. In our experiments, we instead utilized fully deuterated $\mathrm{Sul}-\mathrm{Cu}_{2} \mathrm{Cl}_{4}$ single crystals, and put emphasis on cold neutron measurements. Most data were taken using ten to 15 single crystals, with total masses of 1.5-2.5 g, coaligned to an irregular cumulative mosaic of $3.5^{\circ}$. Inelastic data were collected on the SPINS cold-neutron three-axis spectrometer at NCNR, with $E_{f}=3.7 \mathrm{meV}$ neutrons, a cooled $\mathrm{BeO}$ filter after the sample, and a horizontal focusing pyrolitic graphite (PG) analyzer (Setup I). Additional data were taken using the IN14 spectrometer at ILL, in a similar configuration (Setup II). Measurements with an enhanced wave vector resolution were performed on the V2 three-axis spectrometer at HMI, with a narrow focused PG analyzer and a smaller $\sim 1$ g sample with a symmetric triangular mosaic spread of $1.9^{\circ}$ (Setup III). Time-of-flight (TOF) experiments were performed at the Disc Chopper Spectrometer (DCS) at NCNR using a fixed incident energy $E_{i}=6.67 \mathrm{meV}$ (Setup IV).

A survey of the reciprocal space by a series of constant- $q$ and constant $-E$ scans revealed the inadequacy of the original model for $\mathrm{Sul}_{-} \mathrm{Cu}_{2} \mathrm{Cl}_{4}$. Figure 2 shows the energy- $l$ projection of the TOF spectrum measured at $T=70 \mathrm{mK}$. It is immediately apparent that the $1 \mathrm{D}$ AF zone center, where the spin gap $\Delta$ is observed as a minimum in the magnetic excitation spectrum, is located near half-integer values of $l$. No low-energy magnetic scattering was detected at integer $l$. This behavior indicates that it is the

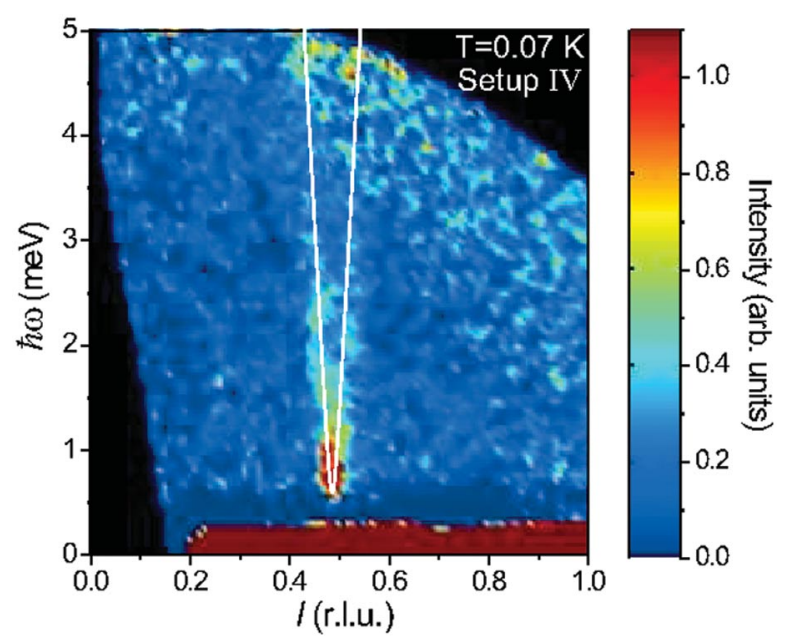

FIG. 2 (color). Time-of-flight spectrum measured in Sul- $\mathrm{Cu}_{2} \mathrm{Cl}_{4}$ in projection onto the $c^{*}$ axis. The solid line is the fitted magnon dispersion relation, as described in the text. next-nearest-neighbor (NNN) spins in each chain which are strongly antiferromagnetically correlated, not the nearest-neighbor ones as proposed in Refs. [14,15]. The magnetic interactions responsible for strong NNN in-chain AF coupling are readily identified. They are established by superexchange across two-chlorine bridges, such as $\mathrm{Cu}_{1}-\mathrm{Cl}-\mathrm{Cl}-\mathrm{Cu}_{1}$ or $\mathrm{Cu}_{2}-\mathrm{Cl}-\mathrm{Cl}-\mathrm{Cu}_{2}$. As was previously found in the structurally related material IPA- $\mathrm{CuCl}_{3}[10]$, and in the Ni-based compound $\mathrm{NiCl}_{2}-4 \mathrm{SC}\left(\mathrm{NH}_{2}\right)_{2}$ [20], $\mathrm{Cu}-$ $\mathrm{Cl}-\mathrm{Cl}-\mathrm{Cu}$ couplings can be much stronger than those across single-halide $\mathrm{Cu}-\mathrm{Cl}-\mathrm{Cu}$ bridge due to more favorable bonding angles and orbital overlap. Thus, each alternating spin chain in $\mathrm{Sul}-\mathrm{Cu}_{2} \mathrm{Cl}_{4}$ should actually be considered as a pair of superimposed AF spin chains, each with a uniform (nonalternating) bond length equal to $c$. Such spin chains are highlighted by bold or dashed $\mathrm{Cu}-\mathrm{Cl}-\mathrm{Cl}-\mathrm{Cu}$ bonds in Fig. 5(a).

Our neutron data indicate that $\mathrm{Sul}-\mathrm{Cu}_{2} \mathrm{Cl}_{4}$ is indeed an exceptionally one-dimensional magnetic material. The dispersion of magnetic excitations is practically absent along the $a^{*}$ and $b^{*}$ axes [Figs. 3(a) and 3(b)], but very steep along the $c^{*}$ direction (Fig. 2). Only the highest-resolution Setup III allowed us to estimate the corresponding spin wave velocity. Typical constant- $E$ scans are shown in Fig. 3(c). At $\hbar \omega=0.6 \mathrm{meV}$, just above the gap energy, resolution-limited peaks are observed. However, at $2.5 \mathrm{meV}$ energy transfer, an intrinsic l-width becomes
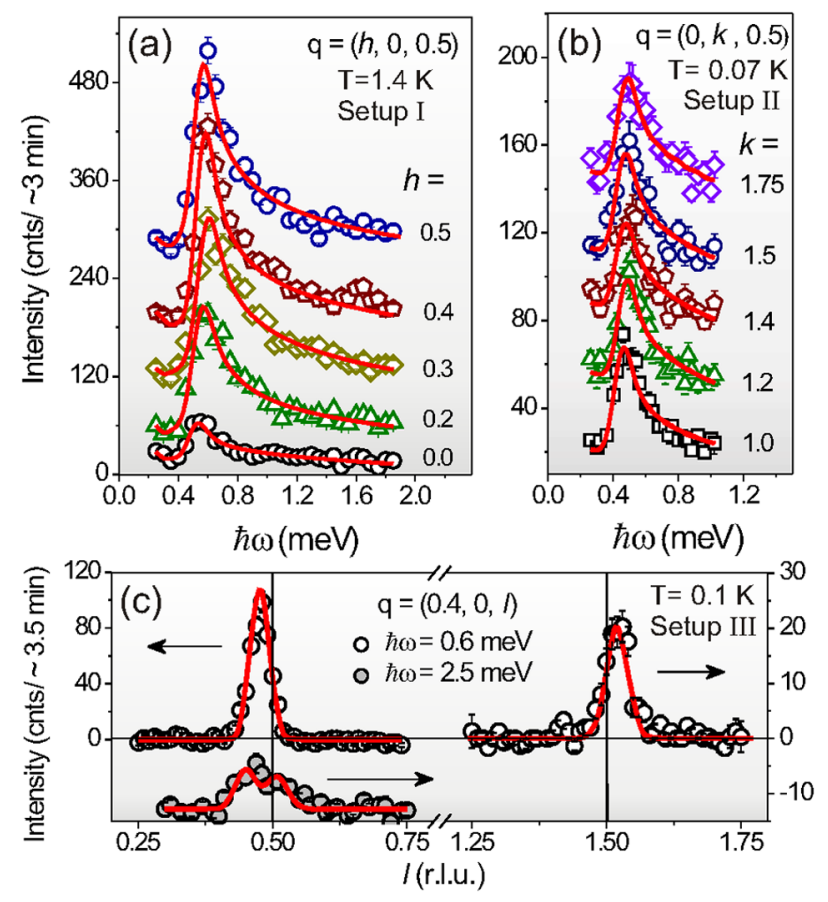

FIG. 3 (color online). (a),(b) Energy scans performed near the 1D AF zone center for different transverse wave vectors. (c) Constant-energy scans along the $l$ direction measured just above the gap energy. Solid lines are fits to the data as described in the text. 
apparent and is due to a finite magnon velocity. As will be discussed below, the peaks are actually centered at incommensurate zone-centers $l_{0}=0.5-\delta$ and $l_{0}=1.5+\delta$, $\delta=0.022(2)$. The approximate dispersion relation for low-energy magnons is therefore written as

$$
\left(\hbar \omega_{\mathbf{q}}\right)^{2}=\Delta^{2}+v^{2}\left(\mathbf{q} \cdot \mathbf{c}-2 \pi l_{0}\right)^{2} .
$$

A simple model cross section function can then be constructed using the single-mode approximation

$$
\frac{d^{2} \sigma}{d \Omega d E^{\prime}} \propto|f(\mathbf{q})|^{2} \frac{|F(\mathbf{q})|^{2}}{\hbar \omega_{\mathbf{q}}} \delta\left(\omega-\omega_{\mathbf{q}}\right)
$$

where $F(\mathbf{q})$ is the a priori unknown structure factor that is expected to be a smooth function of $\mathbf{q}$, and $f(q)$ is the magnetic form factor for $\mathrm{Cu}^{2+}$. Equation (2) convoluted with the known spectrometer resolution function was fit to the bulk of the three-axis data to determine $\Delta=$ $0.52(1) \mathrm{meV}$ and $v=13.7(8) \mathrm{meV}$. Excellent fits are obtained and shown in solid lines in Fig. 3. The resulting dispersion curve is plotted over the TOF data in Fig. 2.

Having determined that $\mathrm{Cu}-\mathrm{Cl}-\mathrm{Cl}-\mathrm{Cu}$ pathways dominate in-chain interactions, we now have to consider their role in the interchain coupling. As illustrated in Fig. 1, such bridges link pairs of adjacent $\mathrm{Cu}^{2+}$ chains in a plane perpendicular to the $b$ axis. Note that these are not the same chain pairs as those considered in Ref. [14]. Recalling that each structural $\mathrm{Cu}^{2+}$ chain actually consists of two superimposed Heisenberg spin chains formed by $\mathrm{NNN}$ AF interactions, we find that $\mathrm{Cu}-\mathrm{Cl}-\mathrm{Cl}-\mathrm{Cu}$ bridges in $\mathrm{Sul}-\mathrm{Cu}_{2} \mathrm{Cl}_{4}$ establish 4-leg spin "ladders," as shown in Fig. 5(a). In this representation, $J_{1}$ denotes the leg coupling responsible for a strong $c$-axis dispersion of magnetic excitations. In addition, three distinct "rung" interactions $J_{2}, J_{3}$, and $J_{4}$ can be identified. None of these are affected by the bond alternation between the two distinct sites, $\mathrm{Cu} 1$ and $\mathrm{Cu} 2$. The four exchange pathways give $\mathrm{Sul}-\mathrm{Cu}_{2} \mathrm{Cl}_{4}$ the unique topology of a 4-leg spin tube, as shown in Fig. 5(b).

The interactions $J_{2}-J_{4}$ establish correlations between the individual legs of each spin tube. Because of the resulting interference effect, the intensity of the gap mode becomes dependent on momentum transfer perpendicular to the chain axis. A constant- $E$ scan taken using Setup I just above the gap energy along the $(h, 0,0.5)$ direction, near the 1D zone-center, is shown in Fig. 4 (open circles) and reveals a strong intensity modulation. The solid symbols represent integrated intensities measured in $l$-scans of the type shown in Fig. 3(c). The observed modulation is periodic, yet off-centered relative to $h=0$. Similar behavior is seen at $(h, 0,1.5)$. To explain this phenomenon quantitatively, we recall that in a simple 2leg spin ladder, the gap modes correspond to spins fluctuating coherently on the two ladder legs [2]. Their intensity is modulated by the modulus-squared of the rung's structure factor. For AF rungs defined by a vector $\mathbf{d},|F(\mathbf{q})|^{2}=$ $\sin ^{2}(\mathbf{q} \cdot \mathbf{d} / 2)$. By analogy, the lowest-energy excitation of the 4-leg spin tube in $\mathrm{Sul}-\mathrm{Cu}_{2} \mathrm{Cl}_{4}$ is a coherent fluctuation of its four legs. The relative phases of the legs will depend on the details of the rung coupling. The intensity of the gap mode becomes modulated by the structure factor of the rung unit comprised of four spins, one on each leg:

$$
F(\mathbf{q})=\sum_{j=1}^{4} \phi_{j} \exp \left(-i \mathbf{q} \cdot \mathbf{r}_{j}\right)
$$

Here, $j$ labels the spins on the rung unit, and $\phi_{j}= \pm 1$ are their corresponding phases. Since in our model interleg correlations are established by $J_{2}, J_{3}$, and $J_{4}$, only those spins directly connected by these interactions need be considered as members of a rung unit. Having tried all possible site and phase combinations, we found that only one can reproduce the measured transverse intensity modulation. The thus identified rung-unit is shown in Fig. 5(a) and coincides with the nominal crystallographic unit cell. The corresponding phases of its four spins are indicated by "+" and "-" signs. This model, with ionic magnetic form factors and resolution effects taken into account, reproduces the observed intensity modulation almost perfectly, as shown in a solid line in Fig. 4. The 4-leg topology may also be responsible for the relatively small excitation gap in $\mathrm{Sul}-\mathrm{Cu}_{2} \mathrm{Cl}_{4}[21,22]$.

Our model naturally accounts for the observed shift of the $1 \mathrm{D}$ AF zone-center relative to the commensurate position. We note that the diagonal AF interaction $J_{4}$ incurs a geometric frustration relative to $J_{1}-J_{3}$ [Fig. 5(b)]. In classical magnets, such frustration induces a helimagnetic state [23]. Theory predicts that in a frustrated quantum 2-leg spin ladder, this incommensurability may survive in the equal-time spin correlation function [24], even though the ground state is disordered. In this case, the minimum of the magnon dispersion occurs at an incommensurate wave vector. The situation in $\mathrm{Sul}-\mathrm{Cu}_{2} \mathrm{Cl}_{4}$ is a direct analogue of this effect in a spin tube with frustrating diagonals. Such a singlet ground state with incommensurate correlations has not been found in any previously studied 1D spin gap system.

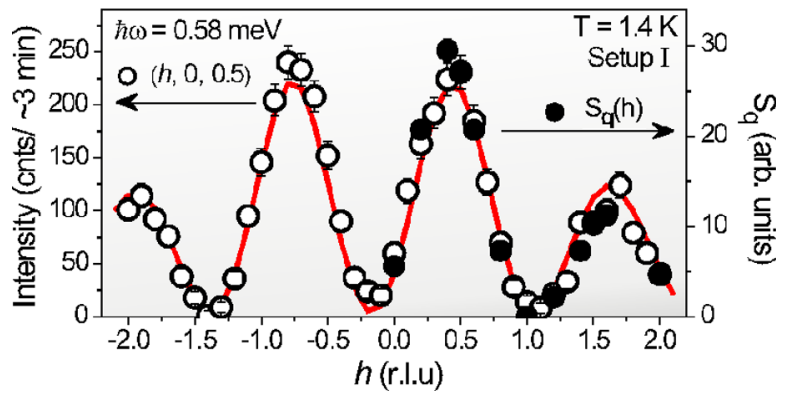

FIG. 4 (color online). Measured $h$-modulation of the scattering intensity of the energy-gap mode at $T=1.4 \mathrm{~K}$. Symbols and lines are as described in the text. 


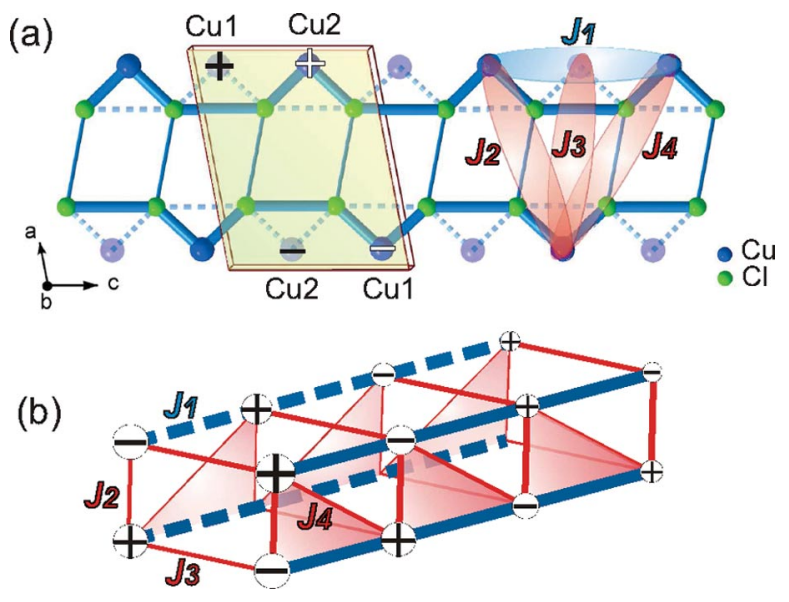

FIG. 5 (color). (a) Ladder-like structure consisting of $\mathrm{Cu}^{2+}$ ions coupled through double chlorine bridges in $\mathrm{Sul}-\mathrm{Cu}_{2} \mathrm{Cl}_{4}$. (b) A topologically equivalent uniform 4-leg spin tube structure. The relative instantaneous orientations of the spins are indicated by "+" and "-" signs.

We conclude that $\mathrm{Sul}-\mathrm{Cu}_{2} \mathrm{Cl}_{4}$ realizes the rare 4-leg spin tube model. The mapping is actually quite accurate: it is not at all perturbed by the intrinsic structural modulation of the $\mathrm{Cu}^{2+}$ chains, nor by three-dimensional interactions that are negligible. The spin tube Hamiltonian posses unique quantum mechanical properties, due to periodic boundary conditions along the interchain direction. However, for previous lack of prototype materials, only a limited number of theoretical results have been accumulated [25-27]. Future work on $\mathrm{Sul}-\mathrm{Cu}_{2} \mathrm{Cl}_{4}$ should focus on higher-energy excitations, where one can expect to find exotic states with higher values of the transverse discrete quantum number. Magnetic field experiments will probe Bose-Einstein condensation of magnons. Because of geometric frustration, one can expect a unique helimagnetic high-field ordered phase.

Research at ORNL was funded by the United States Department of Energy, Office of Basic Energy SciencesMaterials Science, under Contract No. DE-AC0500OR22725 with UT-Battelle, LLC. The work at NCNR was supported by the National Science Foundation under Agreement Nos. DMR-9986442, DMR-0086210, and DMR-0454672. The authors are grateful to Drs. B. Sales and D. Mandrus for making available their laboratory for the sample preparation. We also thank Drs. K. Hradil, P. Böni, and R. Mole for their help in the preliminary INS experiments on the three-axis spectrometer PUMA, at FRM II, Germany.

*garleao@ornl.gov

Currently at: Department of Physics, Korea University, Seoul 136-701, Korea
[1] T. M. Rice, S. Gopalan, and M. Sigrist, Europhys. Lett. 23, 445 (1993).

[2] T. Barnes, E. Dagotto, J. Riera, and E. S. Swanson, Phys. Rev. B 47, 3196 (1993).

[3] S. Gopalan, T. M. Rice, and M. Sigrist, Phys. Rev. B 49, 8901 (1994).

[4] T. Barnes and J. Riera, Phys. Rev. B 50, 6817 (1994).

[5] M. den Nijs and K. Rommelse, Phys. Rev. B 40, 4709 (1989).

[6] T. Kennedy and H. Tasaki, Phys. Rev. B 45, 304 (1992).

[7] O.P. Sushkov and V. N. Kotov, Phys. Rev. Lett. 81, 1941 (1998).

[8] T. Giamarchi and A. M. Tsvelik, Phys. Rev. B 59, 11398 (1999).

[9] A. Furusaki and S.-C. Zhang, Phys. Rev. B 60, 1175 (1999).

[10] T. Masuda, A. Zheludev, H. Manaka, L.-P. Regnault, J.-H. Chung, and Y. Qiu, Phys. Rev. Lett. 96, 047210 (2006).

[11] V. O. Garlea, A. Zheludev, T. Masuda, H. Manaka, L.-P. Regnault, E. Ressouche, B. Grenier, J.-H. Chung, Y. Qiu, K. Habicht, K. Kiefer, and M. Boehm, Phys. Rev. Lett. 98, 167202 (2007); see also A. Zheludev et al., Phys. Rev. B 76, 054450 (2007).

[12] S. Notbohm, P. Ribeiro, B. Lake, D. A. Tennant, K. P. Schmidt, G. S. Uhrig, C. Hess, R. Klingeler, G. Behr, B. Büchner, M. Reehuis, R. I. Bewley, C. D. Frost, P. Manuel, and R. S. Eccleston, Phys. Rev. Lett. 98, 027403 (2007).

[13] M. Matsuda, K. Katsumata, R. S. Eccleston, S. Brehmer, and H.-J. Mikeska, Phys. Rev. B 62, 8903 (2000).

[14] M. Fujisawa, J.-I. Yamaura, H. Tanaka, H. Kageyama, Y. Narumi, and K. Kindo, J. Phys. Soc. Jpn. 72, 694 (2003).

[15] M. Fujisawa, H. Tanaka, and T. Sakakibara, Prog. Theor. Phys. Suppl. 159, 212 (2005).

[16] K. Hirota, D. E. Cox, J. E. Lorenzo, G. Shirane, J. M. Tranquada, M. Hase, K. Uchinokura, H. Kojima, Y. Shibuya, and I. Tanaka, Phys. Rev. Lett. 73, 736 (1994).

[17] L. P. Regnault, M. Aïn, B. Hennion, G. Dhalenne, and A. Revcolevschi, Phys. Rev. B 53, 5579 (1996).

[18] J. Eckert, D. E. Cox, G. Shirane, S. A. Friedberg, and H. Kobayashi, Phys. Rev. B 20, 4596 (1979).

[19] M. Fujisawa, A. Oosawa, H. Tanaka, and K. Kakurai, JAERI experimental report (unpublished).

[20] V. S. Zapf, D. Zocco, B. R. Hansen, M. Jaime, N. Harrison, C. D. Batista, M. Kenzelmann, C. Niedermayer, A. Lacerda, and A. Paduan-Filho, Phys. Rev. Lett. 96, 077204 (2006).

[21] S. R. White, R. M. Noack, and D. J. Scalapino, Phys. Rev. Lett. 73, 886 (1994).

[22] B. Frischmuth, B. Ammon, and M. Troyer, Phys. Rev. B 54, R3714 (1996).

[23] A. Yoshimori, J. Phys. Soc. Jpn. 14, 807 (1959).

[24] S. R. White and I. Affleck, Phys. Rev. B 54, 9862 (1996).

[25] M. Sato and M. Oshikawa, Phys. Rev. B 75, 014404 (2007).

[26] J. Schnack, H. Nojiri, P. Kögerler, G. J. T. Cooper, and L. Cronin, Phys. Rev. B 70, 174420 (2004).

[27] J.-B. Fouet, A. Läuchli, S. Pilgram, R. M. Noack, and F. Mila, Phys. Rev. B 73, 014409 (2006). 